\title{
Photospheric activity of the Sun with VIRGO and GOLF Comparison with standard activity proxies
}

\author{
D. Salabert ${ }^{1,2}$, R. A. García ${ }^{1,2}$, A. Jiménez ${ }^{3,4}$, L. Bertello ${ }^{5}$, E. Corsaro ${ }^{1,2,3,4,6}$, and P. L. Pallé 3,4 \\ 1 IRFU, CEA, Université Paris-Saclay, 91191 Gif-sur-Yvette, France \\ e-mail: david.salabert@cea.fr \\ 2 Université Paris Diderot, AIM, Sorbonne Paris Cité, CEA, CNRS, 91191 Gif-sur-Yvette, France \\ 3 Instituto de Astrofísica de Canarias, 38200 La Laguna, Tenerife, Spain \\ 4 Departamento de Astrofísica, Universidad de La Laguna, 38205 La Laguna, Tenerife, Spain \\ 5 National Solar Observatory, 3665 Discovery Drive, Boulder, CO 80303 , USA \\ ${ }^{6}$ INAF-Observatorio Astrofisico di Catania, via S. Sofia 78, 95123 Catania, Italy
}

Received 13 July 2017 / Accepted 14 September 2017

\begin{abstract}
We study the variability of solar activity using new photospheric proxies originally developed for the analysis of stellar magnetism with the CoRoT and Kepler photometric observations. These proxies were obtained by tracking the temporal modulations in the observations associated with the spots and magnetic features as the Sun rotates. We analyzed $21 \mathrm{yr}$ of observations, spanning solar cycles 23 and 24, collected by the space-based photometric VIRGO and radial velocity GOLF instruments on board the SoHO satellite. We then calculated the photospheric activity proxy $S_{\text {ph }}$ is for each of the three VIRGO photometers and the associated $S_{\text {vel }}$ proxy from the radial velocity GOLF observations. Comparisons with several standard solar activity proxies sensitive to different layers of the Sun demonstrate that these new activity proxies, $S_{\mathrm{ph}}$ and $S_{\text {vel }}$, provide a new manner to monitor solar activity. We show that both the long-and short-term magnetic variabilities respectively associated with the 11-yr cycle and the quasi-biennial oscillation are well monitored, and that the magnetic field interaction between the subsurface, photosphere, and chromosphere of the Sun was modified between Cycle 24 and Cycle 23. Furthermore, the photometric proxies show a wavelength dependence of the response function of the solar photosphere among the three channels of the VIRGO photometers, providing inputs for the study of the stellar magnetism of Sun-like stars.
\end{abstract}

Key words. Sun: activity - methods: observational

\section{Introduction}

The space-based, Sun-as-a-star Variability of Solar Irradiance and Gravity Oscillations (VIRGO; Fröhlich et al. 1995) and Global Oscillations at Low Frequency (GOLF; Gabriel et al. 1995) instruments on board the Solar and Heliospheric Observatory (SoHO; Domingo et al. 1995) satellite were designed to study the internal structure and rotation of the Sun by measuring the low-degree oscillations over the entire disc in intensity and radial velocity, respectively (e.g., in the following early references Turck-Chièze et al. 1997; Fröhlich et al. 1997). The first reliable signatures of the existence of gravity modes in the Sun (and by consequence in Sun-like stars) were also detected with the GOLF instrument (García et al. 2007; Fossat et al. 2017). Moreover, by adding three polarizing elements at the entrance of the instrument, GOLF was able to measure the two circular polarized components of the solar light and thus to estimate the solar mean magnetic field (SMMF). Unfortunately, owing to a malfunction of the motors moving the polarizers, only 26 days of SMMF observations were acquired. From those observations, an average value of $0.120 \pm 0.002 \mathrm{G}$ was determined (García et al. 1999).

Long and continuous helioseismic observations offer a unique opportunity to monitor and study solar activity at different temporal scales. The response of the acoustic oscillations to solar activity, through the variability of the eigenfrequencies, was proven to provide insights into the structural and magnetic changes in the subsurface layers of the Sun along the solar cycle (see Basu et al. 2012; Salabert et al. 2015, and references therein). However, while the frequency shifts varied with very high levels of correlation with surface activity over the last three solar cycles 21, 22, and 23 (Chaplin et al. 2007), significant differences were observed between frequency shifts and solar activity during the unusual long and deep minimum of Cycle 23 (Salabert et al. 2009; Tripathy et al. 2010) related to magnetically weaker shallow layers of the Sun. In addition, a faster temporal modulation of the acoustic frequency shifts with a period of about two years was also observed (Fletcher et al. 2010). This quasi-biennial oscillation (QBO) is measured in various activity proxies (see, e.g., Bazilevskaya et al. 2015, and references therein) and is characterized by intermittence in periodicity and with amplitudes that vary with time, being is largest at solar maximum. In addition, the other acoustic parameters, such as mode powers and lifetimes, were also observed to vary with magnetic activity in relation with the 11-yr solar cycle (Chaplin et al. 2000; Salabert et al. 2003).

Space-based photometric and radial velocity helioseismic observations provide yet another unexplored way to monitor solar activity. In the stellar context, García et al. (2010) showed, 

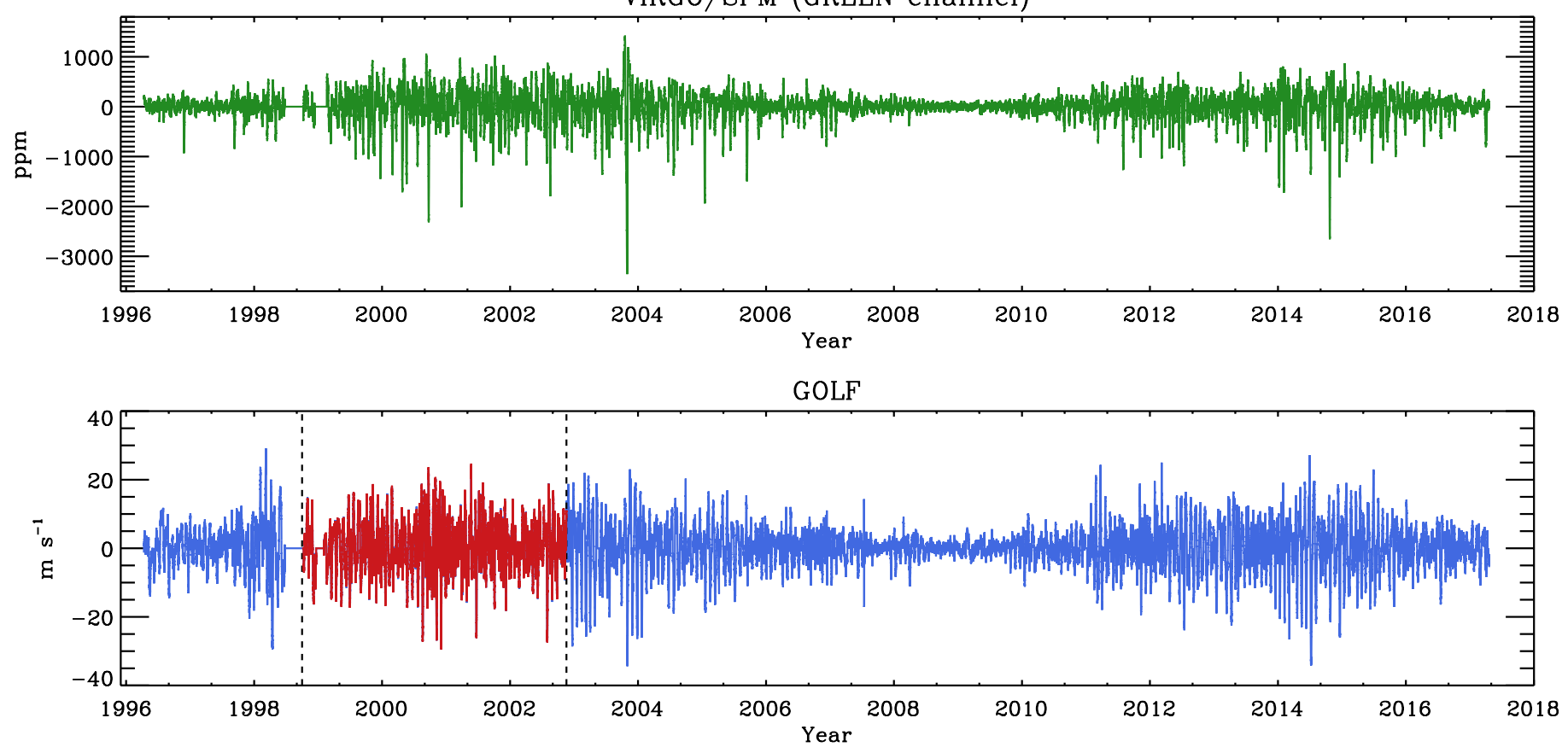

Fig. 1. Space-based observations of the Sun collected by the photometric VIRGO/SPM (top panel, here the GREEN channel) and the radial velocity GOLF (bottom panel) instruments on board the SoHO satellite between 1996 April 11 and 2017 April 11. The missing data shown at zero between the years 1998 and 1999 correspond to the two temporary losses of SoHO. The GOLF red-wing period from mid-1998 to the end of 2002, denoted by the two vertical dashed lines, is represented in red (see Sect. 2).

in the case of the F-star HD 49933 observed with the Convection, Rotation, and planetary Transits (CoRoT; Baglin et al. 2006) space telescope, that the fluctuations associated with the presence of spots or magnetic features rotating on the surface of the star can be analyzed to derive a global proxy of stellar magnetic activity. Nevertheless, as the variability in the data can have different origins with various timescales, such as convective motions, oscillations, stellar companion, or instrumental problems, the rotation period of the star needs to be taken into account in calculating such a magnetic activity proxy. Mathur et al. (2014a) demonstrated that the measured fluctuations estimated as the standard deviations calculated over subseries of length $5 \times P_{\text {rot }}$, where $P_{\text {rot }}$ is the rotation period of the star in days, provide a global proxy only related to magnetism. Since then this global proxy is referred to as the so-called photospheric activity proxy $S_{\mathrm{ph}}$. Initially developed to be applied to the photometric observations collected by the Kepler (Borucki et al. 2010) and CoRoT satellites to study the signature of stellar magnetic activity of main-sequence stars (García et al. 2014; Mathur et al. 2014b; Ferreira Lopes et al. 2015; Salabert et al., in prep.), the $S_{\text {ph }}$ proxy was used to show that the activity of seismic solar analogs is comparable to the Sun within the maximum-to-minimum temporal variations of the 11-yr solar cycle (Salabert et al. 2016). Moreover, Salabert et al. (2016) show the complementarity between the solar photospheric $S_{\mathrm{ph}}$ proxy and the chromospheric activity such as the $\mathrm{Ca} \mathrm{K}$-line emission index. Furthermore, the photospheric $S_{\mathrm{ph}}$ proxy can be easily estimated for a large number of stars with known rotation period from space photometric observations, unlike the standard chromospheric $\mathcal{S}$ index (Wilson 1978) whose estimation requires a large amount of ground-based telescope time to collect enough spectroscopic data for each individual bright target only. The $S_{\text {ph }}$ index is dependent on the inclination angle of the rotation axis in respect to the line of sight and thus provides a lower limit of stellar activity, assuming that the starspots are formed over comparable ranges of latitude as in the Sun. Nevertheless, if the distribution of the spin orientation in space is random, then the most observed inclination angle would be close to $90^{\circ}$, hence perpendicular to the line of sight (see Corsaro et al. 2017). This implies, assuming this hypothesis is verified, that the $S_{\mathrm{ph}}$ index thus measured represents the actual level of activity for most of the stars and not a lower limit.

In this work, we analyzed $21 \mathrm{yr}$ of the space photometric VIRGO and radial velocity GOLF observations to show that such photospheric proxies can be used to monitor solar activity and its long- and short-term temporal evolutions. In Sect. 2, we describe the set of observations used in this analysis. In Sect. 3, we analyze the temporal variations of these photospheric activity proxies over the solar cycles 23 and 24 and we compare such proxies to standard activity proxies. In Sect. 4, we study the wavelength dependence of the photospheric $S_{\mathrm{ph}}$ proxy derived from the VIRGO observations. Conclusions are presented in Sect. 5.

\section{Observations}

The space-based VIRGO and GOLF instruments on board SoHO have been collecting continuous observations of the Sun since the beginning of 1996 with temporal cadences of 60 and $10 \mathrm{~s}$, respectively. However, two temporary losses of the SoHO spacecraft result in two extended gaps in the VIRGO and GOLF acquisition. The first gap of about 100 days happened during the summer of 1998 after a bad maneuver of the SoHO rotation. The second gap, about a month long, occurred in January 1999 while a new software was being uploaded to the spacecraft that caused the SoHO gyroscopes to fail. In this work, we used a total of $21 \mathrm{yr}$ of VIRGO and GOLF data from 1996 April 11 to 2017 April 11 spanning solar cycles 23 and 24 . The associated duty cycles are $94.7 \%$ and $96.6 \%$, respectively. 
The VIRGO instrument is composed of three Sun photometers (SPM) at $402 \mathrm{~nm}$ (BLUE channel), $500 \mathrm{~nm}$ (GREEN channel), and $862 \mathrm{~nm}$ (RED channel). The VIRGO/SPM photometric observations were calibrated as described in Jiménez et al. (2002). In addition, a composite photometric time series was also obtained by combining the observations from the GREEN and RED VIRGO channels, whose combination has the closest bandwidth to the Kepler instrument (see Basri et al. 2010). This COMPOSITE series should be used when comparing the Sun with the stars observed by Kepler.

The GOLF instrument was designed to measure the Doppler wavelength shift integrated over the solar surface in the D1 and D2 Fraunhofer sodium lines at 589.6 and $589.0 \mathrm{~nm}$, respectively. Unfortunately, because of a malfunction in its polarization mechanism, the GOLF instrument has not been working in its nominal configuration since shortly after the launch of SoHO (García et al. 2004). Instead, only one side of the sodium doublet is observed, from which a proxy of the Doppler velocity signal is produced (García et al. 2005). Moreover, the GOLF observations have been collected from each side of the doublet as follows: in the BLUE-wing configuration from 1996 April 11 until 1998 June 24 (i.e., 805 days), and later on from 2002 November 19 until now (the so-called BLUE-wing periods); and in the red-wing configuration of the sodium doublet between 1998 October 30 until 2002 November 18 (i.e., 1481 days; the so-called red-wing period).

The VIRGO/SPM and GOLF instruments are sensitive to the conditions at different heights in the solar atmosphere. In the case of the photometric VIRGO/SPM observations, the response functions of the three channels peak around the base of the photosphere (Fligge et al. 1998), the BLUE and GREEN channels at $-20 \mathrm{~km}$, and the RED channel at $+10 \mathrm{~km}$ (Jiménez et al. 2005). On the other hand, Jiménez-Reyes et al. (2007) showed that the response function of the Doppler velocity GOLF measurements in the sodium Fraunhofer line is different between the BLUEand the red-wing configurations with averaged heights in the solar atmosphere of 322 and $480 \mathrm{~km}$, respectively. Then, the measurements obtained in the red-wing configuration originate higher up in the solar atmosphere with larger contributions from the chromosphere.

In order to monitor the long-lived features on the solar surface, the VIRGO/SPM and GOLF data were processed and highpass filtered through the Kepler Asteroseismic Data Analysis and Calibration Software (KADACS; García et al. 2011). We stress that great care is needed in the choice of the high-pass filter as it affects the absolute values of the measured photospheric activity proxies. Here, a cut-off of 70 days was used, and thus the impact of the filter is within the errors as shown in Salabert et al. (2016). Finally, the VIRGO/SPM and GOLF data were rebinned at $30 \mathrm{~min}$ to mimic the Kepler long-cadence data with a temporal sampling of $29.4244 \mathrm{~min}$. Figure 1 shows the $21 \mathrm{yr}$ of the photometric VIRGO/SPM (GREEN channel) and radial velocity GOLF observations thus obtained and analyzed in this work.

\section{Photometric and velocity activity proxies}

The photospheric activity proxy $S_{\text {ph }}$ was calculated independently for the VIRGO/SPM BLUE, GREEN, and RED channels and for the COMPOSITE data, over subseries of 125 days, corresponding to $5 \times P_{\text {rot }_{\odot}}$, for a solar rotation at the equator $P_{\text {rot }_{\odot}}$ of 25 days. An overlap of 15.625 days between consecutive subseries was also introduced. Only nonzero data points (i.e., no missing data) were used in the computation of $S_{\mathrm{ph}}$. The associated standard errors of the mean values were taken as estimates

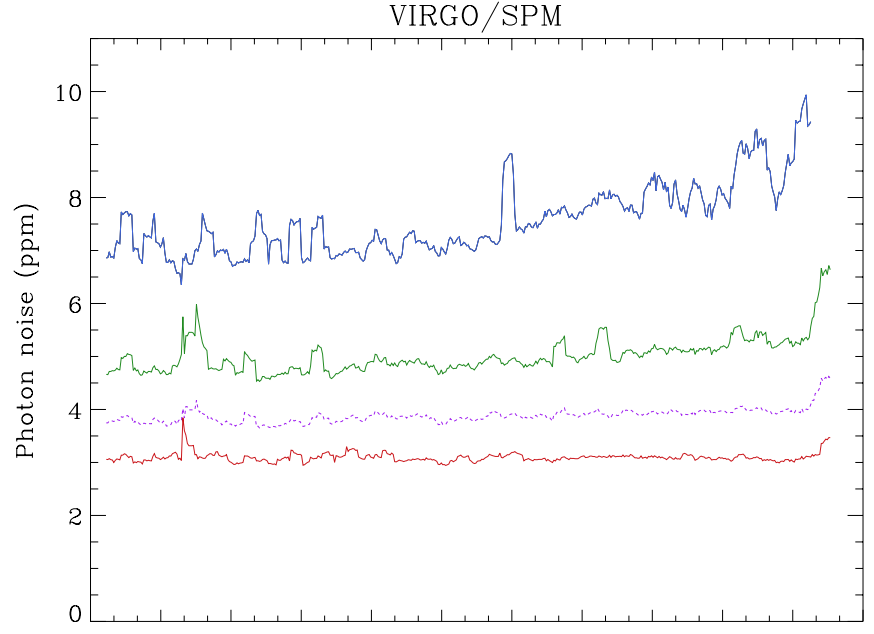

199619982000200220042006200820102012201420162018 Year

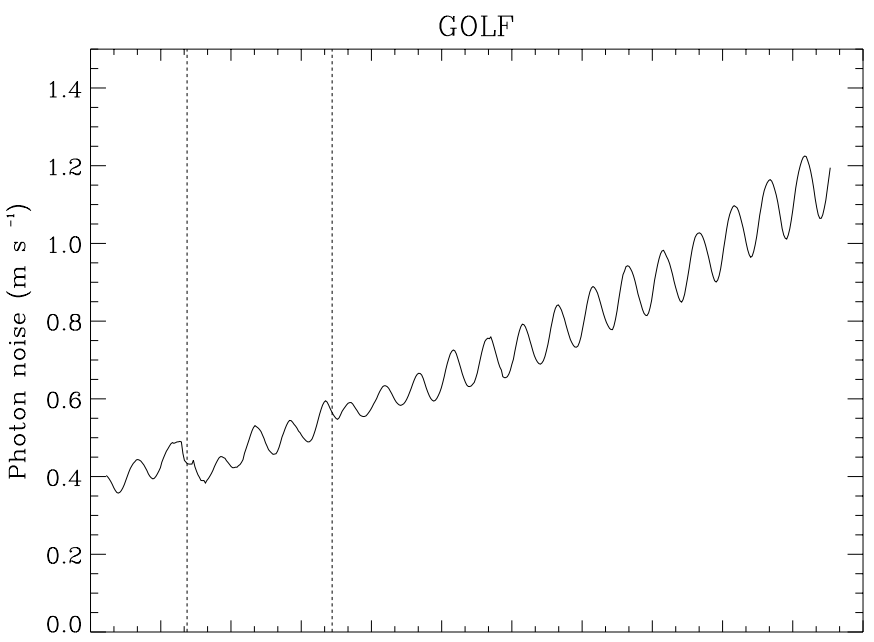

199619982000200220042006200820102012201420162018 Year

Fig. 2. Top panel: photon noise in ppm as a function of time of the photometric VIRGO/SPM observations (color-coded BLUE, GREEN, and RED channels, and coMPosiTE in dotted purple). Bottom panel: same as the top panel but for the radial velocity GOLF observations in $\mathrm{m} \mathrm{s}^{-1}$. The vertical dashed lines indicate the GOLF red-wing period from mid-1998 to the end of 2002.

of the errors on $S_{\mathrm{ph}}$. A similar analysis of the radial velocity GOLF observations provided the so-called $S_{\text {vel }}$ activity proxy.

\subsection{Photon noise correction}

The $S_{\mathrm{ph}}$ and $S_{\text {vel }}$ activity proxies were corrected from the associated photon noise estimated from the high-frequency region (over $8000 \mu \mathrm{Hz}$ ) of the power spectrum of the corresponding observations. Figure 2 shows the photon noise as a function of time for VIRGO/SPM and GOLF. This photon noise was estimated from the unfiltered observations with their original temporal cadence, i.e., 60 and $10 \mathrm{~s}$, respectively (see Sect. 2) to access to the frequency regime of the photon noise with the highest Nyquist frequency possible. In the case of VIRGO, the cadence of $60 \mathrm{~s}$ gives a Nyquist frequency of $8333.33 \mu \mathrm{Hz}$, which is close to the pseudo-mode regime between the acoustic cut-off frequency at $\sim 5500 \mu \mathrm{Hz}$ and about $7500 \mu \mathrm{Hz}$ (García et al. 1998; Jiménez et al. 2005). The power spectrum reaches the Gaussian distributed white noise regime above $8000 \mu \mathrm{Hz}$, which allows us 

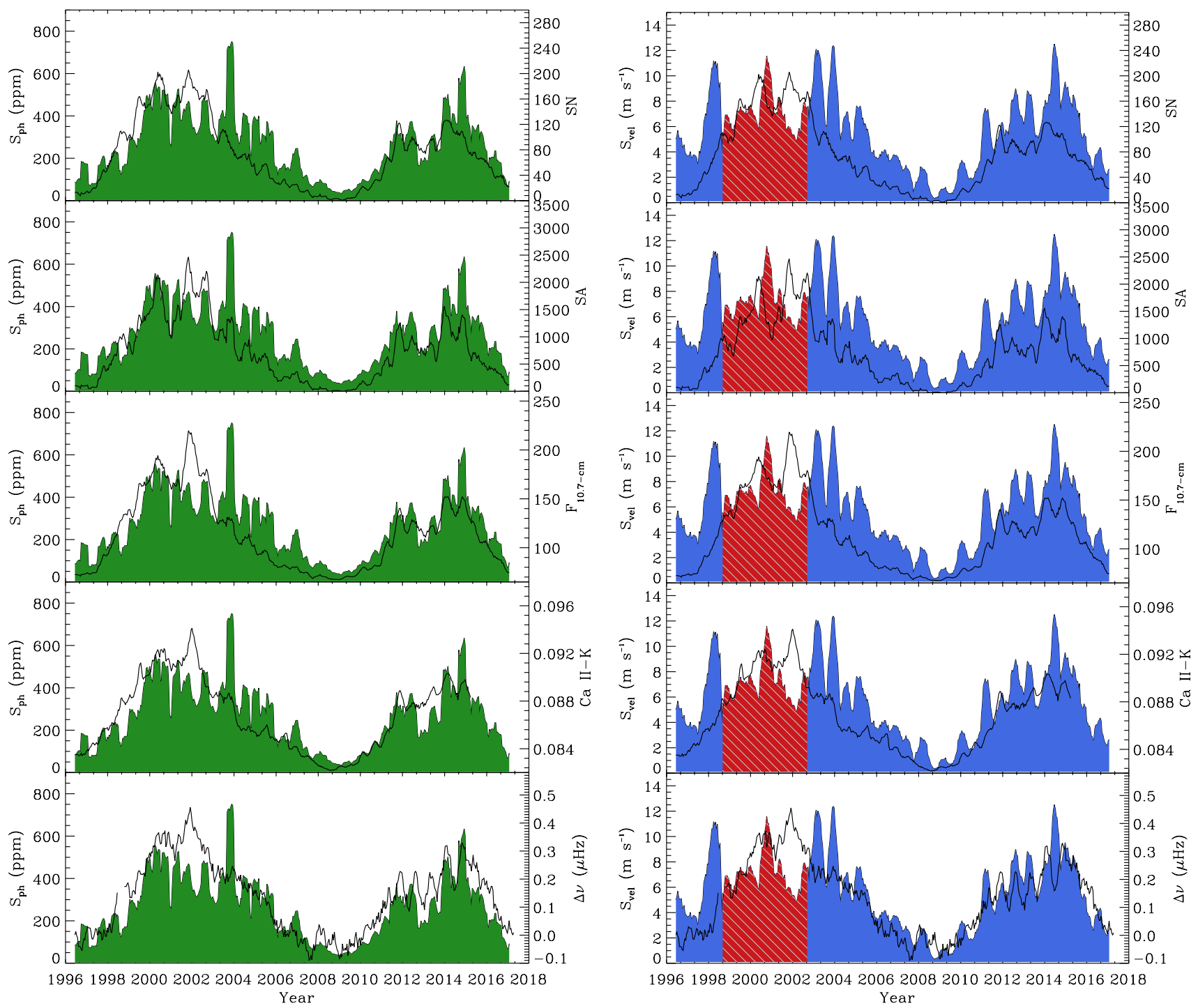

Fig. 3. Magnetic activity proxies $S_{\mathrm{ph}, G R E E N}$ (in ppm) and $S_{\text {vel }}$ (in $\mathrm{m} \mathrm{s}^{-1}$ ) measured from the photometric VIRGO/SPM GREEN channel (GREEN filled, left panels) and the radial velocity GOLF (BLUE filled, right panels) observations as a function of time and compared to standard indices of solar activity (solid black lines). From top to bottom, the total sunspot number $(\mathrm{SN})$, total sunspot area $(\mathrm{SA}), 10.7-\mathrm{cm}$ radio flux $\left(\mathrm{F}_{10.7-\mathrm{cm}}\right)$ in $10^{-22} \mathrm{~s}^{-1} \mathrm{~m}^{-2} \mathrm{~Hz}^{-1}$, Ca II K-line line emission index in $\AA$, and mean frequency shifts of the $l=0,1$ and 2 acoustic oscillations $(\Delta v)$ in $\mu \mathrm{Hz}$ are shown. The red shaded areas in the right-hand panels represent the period when GOLF was observing in the red wing of the sodium line.

to estimate the photon noise up to $8200 \mu \mathrm{Hz}$. We checked that over that frequency range the mean and standard deviation of the power spectrum return similar values. The signature of the data acquisition system (DAS) of the VIRGO instrument with a 3-min cadence at $5555.55 \mu \mathrm{Hz}$ has two important harmonics at $2777.78 \mu \mathrm{Hz}$ in the $p$-mode region and at the $8333.33 \mu \mathrm{Hz}$ Nyquist frequency. For the latter reason, we thus limited the calculations of the VIRGO/SPM photon noise to the upper limit of $8200 \mu \mathrm{Hz}$.

For validation purposes, we have also calculated the VIRGO/SPM photon noise by performing a Bayesian analysis of the background via the DIAMONDS code (Corsaro \& De Ridder 2014). The background profile, $P$, of the unsmoothed power spectra was modeled as described in Corsaro et al. (2015) following:

$P(v)=B(v)+L(v)+N$,

where $B(v)$ includes the contribution of the different timescales of granulation and the signature of activity at low frequency, and $L(v)$ corresponds to the Lorentzian-like power excess envelope of the solar oscillations over the entire $p$-mode region. The variable $N$ represents the flat photon noise at high frequency. The background of asteroseisimic targets is obtained in a similar manner (see, e.g., Kallinger et al. 2014). We compared $N$ to the estimates of the photon noise obtained as the mean value of the power spectra between 8000 and $8200 \mu \mathrm{Hz}$ as explained above. We found that the values of the photon noise thus measured correspond to within $1.0,0.7$, and $0.4 \mathrm{ppm}$ to those inferred from the Bayesian analysis for the BLUE, GREEN, and RED channels, respectively. Although they should be considered as upper limits of the VIRGO/SPM photon noise, the differences are well within the mean errors on the $S_{\mathrm{ph}}$ proxy of 5,4 , and $2 \mathrm{ppm}$, respectively. Furthermore, they represent an easy and reproducible way to obtain the VIRGO/SPM photon noise.

The top panel of Fig. 2 shows that the temporal evolution of the VIRGO/SPM photon noise is different between the three SPM channels. The BLUE channel is noisier and shows a clear increase with time starting from the beginning of the mission 
D. Salabert et al.: Photospheric activity of the Sun with VIRGO and GOLF

Table 1. Spearman's correlation coefficients between the photometric VIRGO/SPM $S_{\text {ph }}$ and the radial velocity GOLF $S_{\text {vel }}$ magnetic proxies and standard indices of solar activity over 21 yr during Cycles 23 and 24.

\begin{tabular}{|c|c|c|c|c|c|c|c|c|c|c|}
\hline Activity proxy & $S_{\text {ph,BLUE }}$ & $S_{\text {ph,GREEN }}$ & $S_{\mathrm{ph}, \mathrm{RED}}$ & $S_{\text {ph,COMPOSITE }}$ & $S_{\mathrm{vel}}$ & $\mathrm{SN}^{a}$ & $\mathrm{SA}^{b}$ & $\mathrm{~F}_{10.7-\mathrm{cm}^{c}}$ & $\mathrm{Ca}$ II K $\mathrm{K}^{d}$ & $\Delta v^{e}$ \\
\hline $\mathrm{SN}^{a}$ & 0.86 & 0.84 & 0.86 & 0.84 & 0.88 & $\mathrm{n} / \mathrm{a}$ & 0.98 & 0.99 & 0.98 & 0.92 \\
\hline $\mathrm{SA}^{b}$ & 0.91 & 0.89 & 0.90 & 0.89 & 0.90 & - & $\mathrm{n} / \mathrm{a}$ & 0.98 & 0.96 & 0.93 \\
\hline $\mathrm{F}_{10.7-\mathrm{cm}^{c}}$ & 0.88 & 0.86 & 0.88 & 0.86 & 0.90 & - & - & $\mathrm{n} / \mathrm{a}$ & 0.98 & 0.94 \\
\hline $\mathrm{Ca}$ II K $\mathrm{K}^{d}$ & 0.87 & 0.85 & 0.86 & 0.85 & 0.87 & - & - & - & $\mathrm{n} / \mathrm{a}$ & 0.93 \\
\hline$\Delta v^{e}$ & 0.89 & 0.87 & 0.89 & 0.88 & 0.86 & - & - & - & - & $\mathrm{n} / \mathrm{a}$ \\
\hline
\end{tabular}

Notes. ${ }^{(a)}$ Total sunspot number; ${ }^{(b)}$ total sunspot area; ${ }^{(c)} 10.7-\mathrm{cm}$ radio flux; ${ }^{(d)}$ Ca II K-line emission index; and ${ }^{(e)}$ mean $l=0,1$, and 2 frequency shifts.

Table 2. Spearman's correlation coefficients between the photometric VIRGO/SPM $S_{\mathrm{ph}}$ magnetic proxies and standard indices of solar activity during Cycle 23.

\begin{tabular}{|c|c|c|c|c|c|c|c|c|c|}
\hline Activity proxy & $S_{\mathrm{ph}, \mathrm{BLUE}}$ & $S_{\text {ph,GREEN }}$ & $S_{\mathrm{ph}, \mathrm{RED}}$ & $S_{\text {ph,COMPOSITE }}$ & $\mathrm{SN}^{a}$ & $\mathrm{SA}^{b}$ & $\mathrm{~F}_{10.7-\mathrm{cm}^{c}}$ & $\mathrm{Ca}$ II K ${ }^{d}$ & $\Delta v^{e}$ \\
\hline $\mathrm{SN}^{a}$ & 0.85 & 0.83 & 0.86 & 0.84 & $\mathrm{n} / \mathrm{a}$ & 0.98 & 0.99 & 0.98 & 0.93 \\
\hline $\mathrm{SA}^{b}$ & 0.89 & 0.88 & 0.90 & 0.88 & - & $\mathrm{n} / \mathrm{a}$ & 0.98 & 0.96 & 0.93 \\
\hline $\mathrm{F}_{10.7-\mathrm{cm}^{c}}$ & 0.86 & 0.84 & 0.87 & 0.84 & - & - & $\mathrm{n} / \mathrm{a}$ & 0.98 & 0.94 \\
\hline $\mathrm{Ca}$ II K ${ }^{d}$ & 0.85 & 0.83 & 0.86 & 0.84 & - & - & - & $\mathrm{n} / \mathrm{a}$ & 0.93 \\
\hline$\Delta v^{e}$ & 0.88 & 0.86 & 0.88 & 0.87 & - & - & - & - & $\mathrm{n} / \mathrm{a}$ \\
\hline
\end{tabular}

Notes. ${ }^{(a)}$ Total sunspot number; ${ }^{(b)}$ total sunspot area; ${ }^{(c)} 10.7$-cm radio flux; ${ }^{(d)}$ Ca II K-line emission index ; and ${ }^{(e)}$ mean $l=0,1$, and 2 frequency shifts.

Table 3. Spearman's correlation coefficients between the photometric VIRGO/SPM $S_{\mathrm{ph}}$ magnetic proxies and standard indices of solar activity during Cycle 24.

\begin{tabular}{cccccccccc}
\hline \hline Activity proxy & $S_{\text {ph,BLUE }}$ & $S_{\text {ph,GREEN }}$ & $S_{\text {ph,RED }}$ & $S_{\text {ph,Composite }}$ & SN $^{a}$ & $\mathrm{SA}^{b}$ & $\mathrm{~F}_{10.7-c m}{ }^{c}$ & $\mathrm{Ca} \mathrm{II} \mathrm{K}^{d}$ & $\Delta v^{e}$ \\
\hline $\mathrm{SN}^{a}$ & 0.92 & 0.88 & 0.86 & 0.87 & $\mathrm{n} / \mathrm{a}$ & 0.98 & 0.97 & 0.93 & 0.86 \\
$\mathrm{SA}^{b}$ & 0.94 & 0.90 & 0.89 & 0.90 & - & $\mathrm{n} / \mathrm{a}$ & 0.97 & 0.93 & 0.86 \\
$\mathrm{~F}_{10.7-\mathrm{cm}}{ }^{d}$ & 0.94 & 0.92 & 0.90 & 0.92 & - & - & $\mathrm{n} / \mathrm{a}$ & 0.96 & 0.91 \\
$\mathrm{Ca} \mathrm{II} \mathrm{K}^{d}$ & 0.89 & 0.89 & 0.83 & 0.87 & - & - & - & $\mathrm{n} / \mathrm{a}$ & 0.94 \\
$\Delta v^{e}$ & 0.87 & 0.88 & 0.87 & 0.89 & - & - & - & - & n/a \\
\hline
\end{tabular}

Notes. ${ }^{(a)}$ Total sunspot number; ${ }^{(b)}$ total sunspot area; ${ }^{(c)} 10.7$-cm radio flux; ${ }^{(d)}$ Ca II K-line emission index; and ${ }^{(e)}$ mean $l=0,1$, and 2 frequency shifts.

for a total of about 30\% between 1996 and 2016, while over the same period the photon noise of the GREEN channel increases by about $10 \%$. On the other hand, the photon noise of the RED channel remains very constant with an overall variation of less than $1 \%$. We interpret these results as a consequence of the degradation of the filters due to the incoming radiation that increases the photon noise level as a function of the wavelength associated with a probable degradation of the detectors. This was verified by looking at the original signals, which show similar behaviors between channels. Also, there is a sharp increase in the photon noise in the three channels from $\sim 2017$, which could indicate a new degradation of the instrument. Future observations will help to understand its origin.

In the case of the GOLF observations, the original temporal sampling of $10 \mathrm{~s}$ (i.e., a Nyquist frequency at $50000.00 \mu \mathrm{Hz}$ ) is short enough to have certain access to the photon noise regime. Because of its single-wing configuration, the GOLF velocity data are sensitive to the photon counting, and thus to the orbital period as we can see on the bottom panel of Fig. 2 with a clear 365-day signature. This dependence is added to the aging of the instrument (García et al. 2005). The configuration changes between BLUE-wing and red-wing observations are also visible as denoted by the vertical dashed lines.
For each VIRGO/SPM and GOLF datasets, the photon noise was thus determined over the same 125-day subseries and subtracted from the associated $S_{\mathrm{ph}}$ and $S_{\mathrm{vel}}$ activity proxies.

\subsection{Long-term variability}

The temporal evolution of the $S_{\mathrm{ph}}$, GREEN and $S_{\text {vel }}$ activity proxies calculated over $21 \mathrm{yr}$ of the photometric VIRGO/SPM GREEN channel and radial velocity GOLF observations, respectively, and covering the solar cycles 23 and 24 are shown on Fig. 3. They follow a clear 11-yr modulation, which is larger at times of maxima. Also, comparable temporal variations are obtained

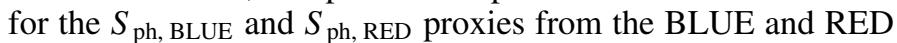
observations, but different sensitivities are observed between the three VIRGO/SPM channels. The wavelength dependence of the $S_{\mathrm{ph}}$ proxy is discussed in Sect. 4. In addition, smaller values of $S_{\text {vel }}$ are obtained when GOLF was observing in the red wing of the sodium line compared to the BLUE wing. This confirms that the red-wing configuration has contributions from regions higher in the solar atmosphere than the BLUE-wing configuration.

In the following, both the $S_{\mathrm{ph}}$ and $S_{\text {vel }}$ proxies are compared to standard solar activity indices, whose daily values were 
averaged over the same 125-day subseries: (1) the total sunspot number ${ }^{1} ;(2)$ the total sunspot area ${ }^{2}$; (3) the 10.7-cm radio flux ${ }^{3}$; and (4) the chromospheric Ca II K-line emission ${ }^{4}$. (5) The photometric and velocity proxies are also compared to the temporal variations of the $l=0,1$, and 2 acoustic oscillation frequencies averaged over the range 2650 and $3450 \mu \mathrm{Hz}$ and extracted from the GOLF observations over the same 125-day subseries following the method described in Salabert et al. (2015). However, the subseries around the temporary losses of SoHO in summer 1998 and beginning of 1999 with a filling factor lower than $50 \%$ were disregarded as the $p$-mode frequencies cannot be estimated with sufficient accuracy. These standard proxies are sensitive to different layers of the Sun. The sunspot number and area are associated with the photospheric magnetic flux, while the radio flux is an activity proxy of the upper chromosphere and lower corona and the Ca II K-line emission a proxy of the strength of plasma emission in the chromosphere. As for the frequency shifts, they reveal inferences on the subsurface changes with solar activity that are not detectable at the surface by other activity proxies. We note as well that Ca II K observations up to 2015 June 10 only were used here because instrumental problems need to be addressed with the latest data. These proxies are compared to $S_{\text {ph }}$ and $S_{\text {vel }}$ in Fig. 3.

Table 1 gives the Spearman's correlation coefficients between the photometric $S_{\mathrm{ph}}$ of the three VIRGO/SPM channels, the associated Kepler-like composite VIRGO/SPM time series, and the GOLF radial velocity $S_{\text {vel }}$ activity proxies and standard proxies monitoring solar activity represented in Fig. 3. The correlations between the common activity indices are given as well for comparison. In the case of GOLF, the correlation coefficients were only calculated for the BLUE-wing period starting on 2002 November 19 to avoid the change between the BLUE and red wings of the sodium line between 1998 and 2002. Also, we only used independent points. Both the $S_{\text {ph }}$ and $S_{\text {vel }}$ proxies are well correlated with the standard solar activity proxies with degrees of correlation around 0.9 with no noticeable difference between the various analyzed datasets.

Figure 4 shows the standard activity proxies illustrated in Fig. 3 as a function of the corresponding photospheric activity index $S_{\mathrm{ph}}$ calculated from the VIRGO/SPM GREEN channel observations. Similar results are obtained with the two other VIRGO/SPM channels. Such representation for the GOLF $S_{\text {vel }}$ is more difficult to interpret because the changes in the side of the observing wings. For illustrative purpose and to remove shortterm variability, the data points were smoothed over a period of $2.5 \mathrm{yr}$. The different colors correspond to different phases in solar activity: in red and BLUE, the rising and declining phases of Cycle 23, respectively; and in GREEN and yellow, the rising and declining phases of Cycle 24, respectively. The individual data points before smoothing are represented by gray dots. There is a clear relationship between the photospheric index $S_{\mathrm{ph}}$ and the other activity proxies from one cycle to the next. The $S_{\mathrm{ph}}$ also shows a smaller variation during the weaker Cycle 24 as it observed in the other activity proxies, which is interestingly not as noticeable for the frequency shifts that are sensitive to the sublayers of the Sun. Such differences of behavior

\footnotetext{
1 Source: WDC-SILSO, Royal Observatory of Belgium, Brussels at http://www.sidc.be/silso/datafiles

2 Source: http://solarcyclescience.com/activeregions. html

3 Source: National Geophysical Data Center at http://www.ngdc. noaa.gov/stp/solar/solardataservices.html

4 Source: composite data from NSO/Sacramento Peak and SOLIS/ISS observations (Bertello et al. 2016).
}

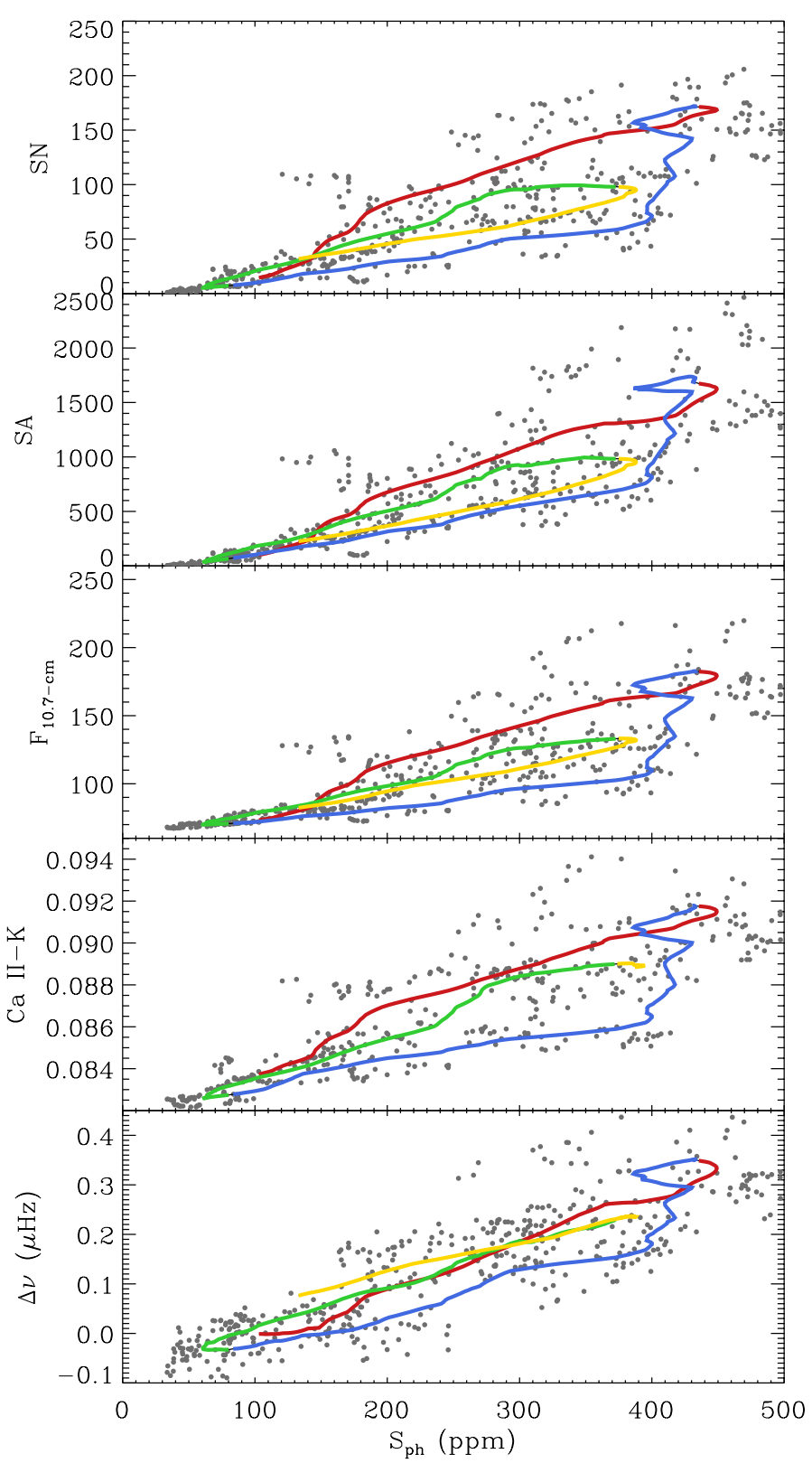

Fig. 4. Standard proxies of solar activity as a function of the photospheric magnetic proxy of the Sun, $S_{\mathrm{ph}, \text { GREEN }}$ (in ppm), measured from the VIRGO/SPM GREEN channel observations. From top to bottom, the $y$-axis represents the sunspot number, sunspot area, $10.7-\mathrm{cm}$ radio flux, Ca II K emission, and mean frequency shifts of the $l=0,1$, and 2 acoustic oscillations. Individual measurements are shown in gray dots and the solid lines represent a smooth over $2.5 \mathrm{yr}$ to remove short-term variations for illustrative purpose. The different colors correspond to different phases in solar activity; in red and BLUE, the rising and declining phases of Cycle 23, respectively; in GREEN and yellow, the rising and declining phases of Cycle 24, respectively, are shown.

of the frequency shifts at the beginning of the new Cycle 24 were already reported in Salabert et al. (2009) and Tripathy et al. (2010). Salabert et al. (2015) also show that these differences are a function of frequency. Indeed, the frequency shifts of the low-frequency modes, which are less sensitive to the upper layers of the Sun, show almost no difference between Cycles 23 and 24 while the frequency shifts of the high-frequency modes are smaller during Cycle 24. Furthermore, Fig. 4 shows that 

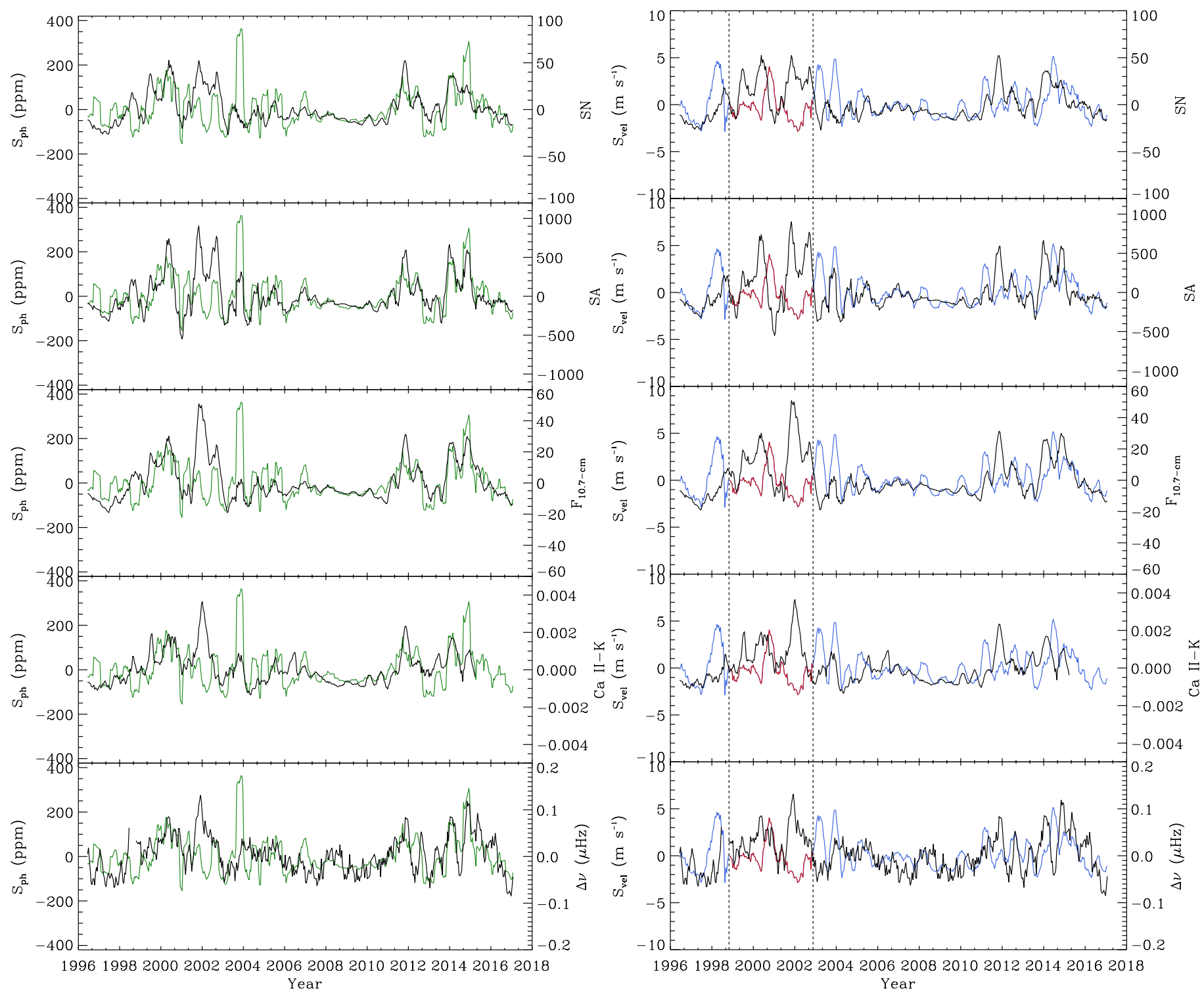

Fig. 5. Same as Fig. 3 but once the signature of the 11-yr solar cycle is removed with a Gaussian filtering (see Sect. 3.3). On the right panels, the two vertical dashed lines indicate the GOLF red-wing period from mid-1998 to the end of 2002.

the relationship between the $S_{\text {ph }}$ proxy and the standard activity proxies is not fully linear and that it follows an hysteresis pattern. Such hysteresis have already been observed between several solar observations of photospheric and chromospheric activity (see, e.g., Bachmann \& White 1994; Özgüç et al. 2012) and with frequency shifts (see, e.g., Jiménez-Reyes et al. 1998; Tripathy et al. 2001). Recently, Salabert et al. (2016) have already shown the existence of such hysteresis between the $S_{\mathrm{ph}}$ and $\mathrm{Ca} \mathrm{K}$-line emission. Time delays are thus clearly present among the various solar activity proxies associated with the distribution in latitude of the surface magnetic flux and its temporal evolution.

Finally, we checked whether any differences can be measured between Cycle 23 and the weaker Cycle 24. Again, we focused here on $S_{\text {ph }}$ from VIRGO observations as the results with the GOLF $S_{\text {vel }}$ between Cycles 23 and 24 are more difficult to interpret because the change in the operation of the instrument throughout the mission. The obtained correlation coefficients are given in Tables 2 and 3. As in Table 1, independent points only were used in the calculations. The $S_{\mathrm{ph}}$ proxies for the BLUE and GREEN channels are observed to be better correlated with the sunspot number, sunspot area, radio flux, and Ca II K emission during Cycle 24 than during Cycle 23. On the other hand, the $S_{\text {ph }}$ proxy for the RED channel does not show differences between the two solar cycles. This could indicate changes in the photospheric response of these activity proxies during Cycle 24 and not in the chromospheric response because the sensitivity of the BLUE and GREEN VIRGO channels peaks below the photosphere. Besides, correlations between standard activity proxies are comparable during the two last solar cycles, except with the $p$-mode frequency shifts and somehow the $\mathrm{Ca}$ II K emission. Indeed, the frequency shifts show a lower degree of correlation with the sunspot number, sunspot area, radio flux, and Ca II K during Cycle 24 than during Cycle 23, which indicates that the subsurface layers have not changed (see Salabert et al. 2015). To summarize, Tables 2 and 3 indicate that modifications of the magnetic field interaction with the solar photosphere and chromosphere, but also with the inner upper layers of the Sun, are taking place between the two last solar cycles. 

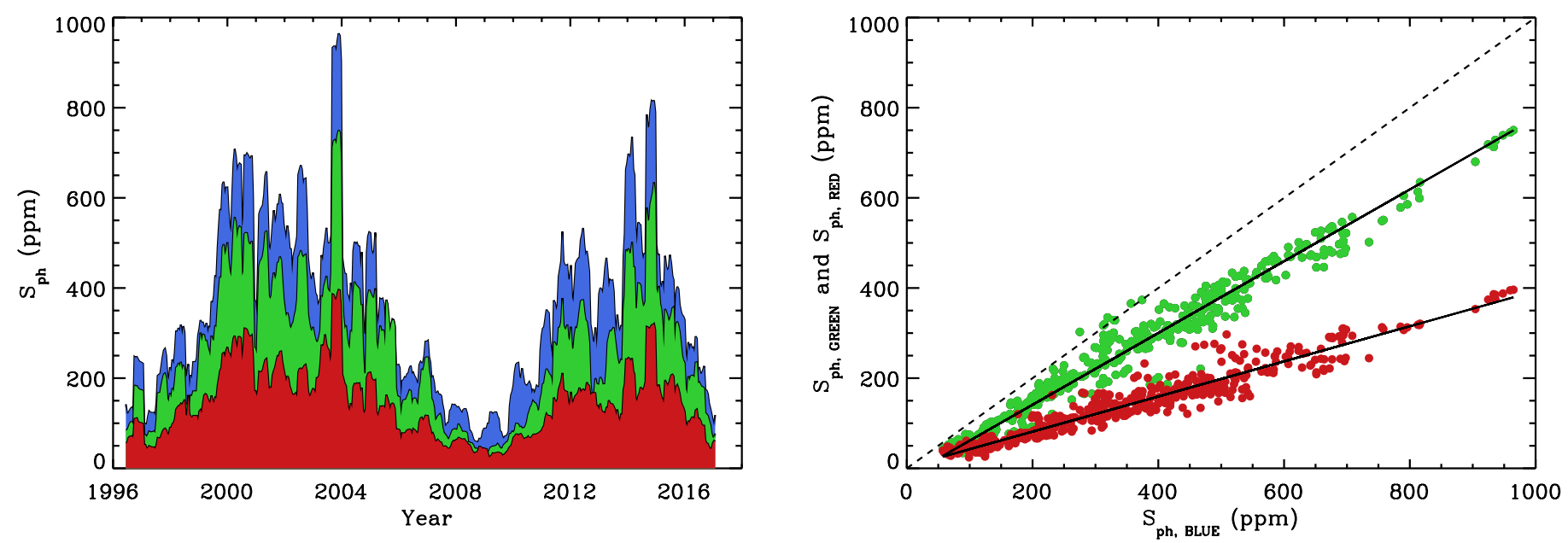

Fig. 6. Left panel: photospheric activity proxies, $S_{\mathrm{ph}}$ (in ppm), for each of the BLUE, GREEN, and RED channels of the VIRGO/SPM observations as a function of time. Right panel: photospheric activity proxy, $S_{\text {ph }}$ from the VIRGO/SPM GREEN (GREEN dots) and RED (red dots) channels as a function of the photospheric index, $S_{\mathrm{ph}}$, from the BLUE channel. The solid lines correspond to weighted linear regressions between the data, and the dashed line represents the 1:1 correlation.

\subsection{Short-term variability}

Short-term variations in the solar activity of about $2 \mathrm{yr}$ (QBO) coexist on top of the 11-yr cycle and are observed by intermittence with time-dependent amplitudes (see, e.g., Bazilevskaya et al. 2015, and references therein). Furthermore, these quasi-periodic variations are observed to be stronger during solar maxima. Figure 5 shows the activity proxies $S_{\mathrm{ph}}$ (GREEN channel) and $S_{\text {vel }}$, and the standard activity proxies once the signature of the 11-yr solar cycle is removed. This was carried out by substracting the corresponding smoothed variations using a Gaussian filter of full width of 2.5-yr length. Shortterm variability exists, which is more or less in phase with the standard solar activity proxies. We observe also stronger shortterm variations in the $S_{\mathrm{ph}}$ and $S_{\mathrm{vel}}$ at time of maxima as observed in other proxies. In addition, the standard proxies are more in phase with $S_{\mathrm{ph}}$ during Cycle 24 than during Cycle 23, which was translated into their degrees of correlation given in Tables 2 and 3 . Regarding the short-term variability of the $p$-mode frequency shifts, we observe a clear change in behavior of the QBO from May 2011. Indeed, a shorter variability of about 270 days is in place for few years during the rising phase of Cycle 24 up to about February 2015 and it disappears during the following declining phase of activity. The solar cycle 24 is clearly peculiar compared to previous cycles and further in-depth analysis of the frequency shifts will be required in order to relate this to any modification of the solar dynamo.

\section{Photometric wavelength dependence to $S_{\mathrm{ph}}$}

The left panel of Fig. 6 shows the photospheric activity proxies $S_{\mathrm{ph}}$ obtained from the three individual BLUE, GREEN, and RED VIRGO/SPM channels as a function of time. While the $S_{\mathrm{ph}}$ is well correlated with solar activity for the three channels, the sensitivity of their variations along the solar cycle is different between channels. Indeed, the BLUE channel shows the largest variations between minimum and maximum of solar activity - of about 600 ppm, while in the mean time the RED channel shows only a variation of about $200 \mathrm{ppm}$. The GREEN channel is closer to the BLUE channel with a variation of about $500 \mathrm{ppm}$ between minimum and maximum of solar activity. The mean uncertainties on $S_{\mathrm{ph}}$ are about 5, 4, and 2 ppm for the BLUE, GREEN, and RED channels, respectively. The largest differences between channels occur at times of solar maxima, while the differences are marginal during solar minima. However, the temporal variations of $S_{\mathrm{ph}}$ between the three channels remain linear, as illustrated on the right panel of Fig. 6, which shows the $S_{\mathrm{ph}}$ proxy from the GREEN and RED channels as a function of the $S_{\mathrm{ph}}$ values from the BLUE channel. The $S_{\mathrm{ph} \text {, RED }}$ has a much reduced amplitude during the phase of maximum of activity, while comparable values are measured between the three channels during the minimum of activity. Regarding the $S_{\mathrm{ph} \text {, GREEN, it has a much }}$ closer temporal behavior to the $S_{\mathrm{ph} \text {, BLUE }}$ channel and to the $1: 1$ correlation. Weighted linear regressions between the quantities give the following relations between the VIRGO/SPM channels:

$S_{\text {ph,GREEN }}=0.756 \times S_{\text {ph,BLUE }}-17.497$

and

$S_{\text {ph,RED }}=0.374 \times S_{\text {ph,BLUE }}+3.653$.

The Eqs. (2) and (3) describe the wavelength dependence of the sensitivity of the $S_{\mathrm{ph}}$ proxy to magnetic activity in photometric observations. Shorter wavelengths, such as that from the VIRGO/SPM BLUE channel at $402 \mathrm{~nm}$, are observed to be more favorable than longer wavelengths, such as the VIRGO/SPM RED channel at $862 \mathrm{~nm}$, to follow solar activity. Fligge et al. (1998) observed comparable relative variations in the irradiance between the three VIRGO channels. Moreover, the values given in Eqs. (2) and (3) are in agreement with those derived by Unruh et al. (1999) with the VIRGO observations of the total and spectral irradiance. Fröhlich et al. (1997) and Jiménez et al. (1999) found comparable ratios as well in the amplitude and the gain, respectively, between the three channels when measuring the acoustic oscillations.

These results can be related to the wavelength dependence of the magnitude of solar variability measured in sunspots. Indeed, the $S_{\text {ph }}$ proxy corresponds to the photometric variations associated with the rotation of spots at the surface of the Sun. Although likely to be more complex, in a first approach, we might suppose that the wavelength dependence of the $S_{\text {ph }}$ proxy is connected to 
the temperature dependence described by Planck's law explaining the larger variations observed at shorter wavelengths than at longer wavelengths. Although the studied range of wavelengths is narrow (from $402 \mathrm{~nm}$ to $862 \mathrm{~nm}$, going from the visible light to the near-infrared), Eqs. (2) and (3) show as a first approximation that a simple linear relation can be used to rescale the activity proxy $S_{\mathrm{ph}}$ obtained at different wavelengths. It thus offers the possibility to compare stellar activity to the Sun measured from different photometric observations of solar-like and mainsequence stars. For instance, the bandpass of the Kepler telescope is comprised between 420 and $900 \mathrm{~nm}$. Although wider, the bandpass of the PLATO (Rauer et al. 2014) space mission should cover a comparable wavelength range as Kepler.

\section{Conclusions}

We analyzed the observations collected by the space-based photometric VIRGO and radial velocity GOLF instruments on board the SoHO satellite in an as yet unexplored way to study the long- and short-term variabilities of solar activity. We tracked the temporal modulations of the observed parameters associated with the presence of spots or magnetic features rotating on the surface of the Sun in a similar manner as performed in the stellar context with CoRoT and Kepler. A total of 21 yr of observations spanning the solar cycles 23 and 24 were thus divided into subseries of $5 \times P_{\text {rot }_{\odot}}=125$ days, with a mean rotational period of the Sun $P_{\text {rot }_{\odot}}$ of 25 days. The associated photospheric activity proxy $S_{\text {ph }}$ was then calculated independently for the photometric VIRGO/SPM BLUE, GREEN, and RED channels and for the combination of the GREEN and RED channels into a COMPOSITE time series. This COMPOSITE dataset has the closest bandwidth to Kepler observations and should be used when comparing the Sun to Kepler targets. The corresponding activity proxy $S_{\text {vel }}$ was also determined from the radial velocity GOLF observations.

Both $S_{\mathrm{ph}}$ and $S_{\text {vel }}$ were compared to several standard solar activity proxies that are sensitive to different layers of the Sun from the inner sublayers up to the corona: the temporal variability of the acoustic oscillation frequencies, sunspot number and sunspot area, Ca II K-line emission, and $10.7-\mathrm{cm}$ radio flux. We showed that these new activity proxies derived from the VIRGO and GOLF observations are well correlated with commonly used activity proxies over the long-term 11-yr variability and that, similar to the other proxies, they indicate as well a weaker $\mathrm{Cy}-$ cle 24 compared to the previous solar cycle. Moreover, these new proxies suggest that modifications of the magnetic field interaction with the solar photosphere and chromosphere, but also with the inner upper layers of the Sun, took place between Cycle 24 and Cycle 23. The short-term variability observed in the Sun, such as the QBO, was also measured to be comparable to other proxies with stronger variations at time of maxima. We demonstrated that these new proxies provide a new manner to monitor solar activity in photometry and radial velocity observations.

Furthermore, the photometric proxies $S_{\text {ph }}$ show a wavelength dependence between the three channels of the VIRGO photometers. The BLUE channel at $402 \mathrm{~nm}$ is the most sensitive to variations with solar activity, while the RED channel at $862 \mathrm{~nm}$ is the least sensitive by a factor 2.7. The GREEN channel at $500 \mathrm{~nm}$ has closer activity sensitivity to the BLUE channel with a factor 1.3. Such observations of the wavelength dependence of the response function of the solar photosphere provide inputs for the study of the stellar magnetism of Sun-like stars. It also provides the opportunity to rescale the activity proxy $S_{\text {ph }}$ of solar-like and main-sequence stars obtained at different wavelengths with the photometric Kepler and PLATO observations and to compare results from these stars to the Sun. These measurements of solar magnetic activity monitoring derived from the VIRGO and GOLF observations are deliverables of the European SpaceInn project and can be found at http: //www . spaceinn.eu.

Acknowledgements. The GOLF and VIRGO instruments on board SoHO are a cooperative effort of many individuals to whom we are indebted. SoHO is a project of international collaboration between ESA and NASA. The authors strongly acknowledge the French space agency, CNES, for its support to GOLF since the launch of SoHO. We are also particularly grateful to Catherine Renaud for her daily check of the GOLF data. D.S. acknowledges the financial support from the CNES GOLF grant and the Observatoire de la Côte d'Azur for support during his stays. E.C. is funded by the European Union's Horizon 2020 research and innovation program under the Marie Sklodowska-Curie grant agreement No. 664931. The research leading to these results has also received funding from the European Community's Seventh Framework Programme ([FP7/2007-2013]) under grant agreement No. 312844 (SPACEINN). The sunspot number data are provided by WDC-SILSO, Royal Observatory of Belgium (Brussels). We thank Solar Cycle Science (http: //solarcyclescience. com) for making their data of the sunspot area freely available. The $10.7-\mathrm{cm}$ radio flux measurements are provided by the National Geophysical Data Center. This work uses SOLIS/ISS data obtained by the NSO Integrated Synoptic Program (NISP) managed by the National Solar Observatory, which is operated by the Association of Universities for Research in Astronomy (AURA), Inc. under a cooperative agreement with the National Science Foundation.

\section{References}

Bachmann, K. T., \& White, O. R. 1994, Sol. Phys., 150, 347

Baglin, A., Michel, E., Auvergne, M., \& COROT Team 2006, Proc. SOHO 18/GONG 2006/HELAS I, Beyond the spherical Sun, 624, 34

Basri, G., Walkowicz, L. M., Batalha, N., et al. 2010, ApJ, 713, L155

Basu, S., Broomhall, A.-M., Chaplin, W. J., \& Elsworth, Y. 2012, ApJ, 758, 43

Bazilevskaya, G., Broomhall, A.-M., Elsworth, Y., \& Nakariakov, V. M. 2015, The Solar Activity Cycle, 53, 359

Bertello, L., Pevtsov, A., Tlatov, A., \& Singh, J. 2016, Sol. Phys., 291, 2967

Borucki, W. J., Koch, D., Basri, G., et al. 2010, Science, 327, 977

Chaplin, W. J., Elsworth, Y., Isaak, G. R., Miller, B. A., \& New, R. 2000, MNRAS, 313, 32

Chaplin, W. J., Elsworth, Y., Miller, B. A., Verner, G. A., \& New, R. 2007, ApJ, 659, 1749

Corsaro, E., \& De Ridder, J. 2014, A\&A, 571, A71

Corsaro, E., De Ridder, J., \& García, R. A. 2015, A\&A, 579, A83

Corsaro, E., Lee, Y.-N., García, R. A., et al. 2017, Nat. Astron., 1, 0064

Domingo, V., Fleck, B., \& Poland, A. I. 1995, Sol. Phys., 162, 1

Ferreira Lopes, C. E., Leão, I. C., de Freitas, D. B., et al. 2015, A\&A, 583, A134

Fletcher, S. T., Broomhall, A.-M., Salabert, D., et al. 2010, ApJ, 718, L19

Fligge, M., Solanki, S. K., Unruh, Y. C., Froehlich, C., \& Wehrli, C. 1998, A\&A, 335,709

Fossat, E., Boumier, P., Corbard, T., et al. 2017, A\&A, 604, A40

Fröhlich, C., Romero, J., Roth, H., et al. 1995, Sol. Phys., 162, 101

Fröhlich, C., Andersen, B. N., Appourchaux, T., et al. 1997, Sol. Phys., 170, 1

Gabriel, A. H., Grec, G., Charra, J., et al. 1995, Sol. Phys., 162, 61

García, R. A., Pallé, P. L., Turck-Chièze, S., et al. 1998, ApJ, 504, L51

García, R. A., Boumier, P., Charra, J., et al. 1999, A\&A, 346, 626

García, R. A., Jiménez-Reyes, S. J., Turck-Chièze, S., \& Mathur, S. 2004, SOHO

14 Helio- and Asteroseismology: Towards a Golden Future, 559, 432

García, R. A., Turck-Chièze, S., Boumier, P., et al. 2005, A\&A, 442, 385

García, R. A., Turck-Chièze, S., Jiménez-Reyes, S. J., et al. 2007, Science, 316, 1591

García, R. A., Mathur, S., Salabert, D., et al. 2010, Science, 329, 1032

García, R. A., Hekker, S., Stello, D., et al. 2011, MNRAS, 414, L6

García, R. A., Ceillier, T., Salabert, D., et al. 2014, A\&A, 572, A34

Jiménez-Reyes, S. J., Régulo, C., Pallé, P. L., \& Roca Cortés, T. 1998, A\&A, 329, 1119

Jiménez, A., Roca Cortés, T., Severino, G., \& Marmolino, C. 1999, ApJ, 525, 1042

Jiménez, A., Roca Cortés, T., Jiménez -Reyes, S. J. 2002, Sol. Phys., 209, 247

Jiménez, A., Jiménez-Reyes, S. J., \& García, R. A. 2005, ApJ, 623, 1215

Jiménez-Reyes, S. J., Chaplin, W. J., Elsworth, Y., et al. 2007, ApJ, 654, 1135 
A\&A 608, A87 (2017)

Kallinger, T., De Ridder, J., Hekker, S., et al. 2014, A\&A, 570, A41 Mathur, S., Salabert, D., García, R. A., \& Ceillier, T. 2014a, J. Space Weather Space Clim., 4, 9

Mathur, S., García, R. A., Ballot, J., et al. 2014b, A\&A, 562, A124

Özgüç, A., Kilcik, A., \& Rozelot, J. P. 2012, Sol. Phys., 281, 839

Rauer, H., Catala, C., Aerts, C., et al. 2014, Exp. Astron., 38, 249

Salabert, D., Jiménez-Reyes, S. J., \& Tomczyk, S. 2003, A\&A, 408, 729

Salabert, D., García, R. A., Pallé, P. L., \& Jiménez-Reyes, S. J. 2009, A\&A, 504, L1
Salabert, D., García, R. A., \& Turck-Chièze, S. 2015, A\&A, 578, A137 Salabert, D., García, R. A., Beck, P. G., et al. 2016, A\&A, 596, A31 Tripathy, S. C., Kumar, B., Jain, K., \& Bhatnagar, A. 2001, Sol. Phys., 200,

Tripathy, S. C., Jain, K., Hill, F., \& Leibacher, J. W. 2010, ApJ, 711, L84 Turck-Chièze, S., Basu, S., Brun, A. S., et al. 1997, Sol. Phys., 175, 247 Unruh, Y. C., Solanki, S. K., \& Fligge, M. 1999, A\&A, 345, 635

Wilson, O. C. 1978, ApJ, 226, 379 